\title{
Evaluation of pleural diseases with FDG-PET imaging: preliminary report
}

\author{
Th Bury, P Paulus, A Dowlati, J L Corhay, P Rigo, M F Radermecker
}

\begin{abstract}
Background - Positron emission tomography (PET) with 18-fluorodeoxyglucose (FDG) is an accurate method for differentiating benign from malignant disease. The use of FDG-PET for the aetiological diagnosis of pleural disease was investigated in 25 patients.

Methods - PET was performed on each subject before invasive procedures were used to determine the aetiological diagnosis. The PET data were analysed by visual interpretation of coronal, sagittal, and transverse slices.

Results - Sixteen patients were found to have malignant pleural disease and nine had benign disease. All patients with histologically confirmed malignant disease showed FDG uptake within the pleural thickening which was intense in 14 cases and moderate in two. PET imaging showed the absence of FDG uptake and correctly categorised seven non-malignant lesions. Two patients with infectious pleural diseases showed a localised and moderate FDG uptake.

Conclusion - Our preliminary results suggest that FDG-PET could be an effective tool for differentiating between benign and malignant pleural diseases.

(Thorax 1997;52:187-189)
\end{abstract}

Keywords: positron emission tomography, fluorodeoxyglucose, pleural disease.

Pleural effusion or pleural thickening are the most common radiographic manifestations of pleural disease but aetiological diagnosis is difficult. Most patients who are admitted to a hospital with pleural disease undergo extensive diagnostic procedures including chest radiography, thoracocentesis with various chemical, microbiological and cytological studies, computed tomographic (CT) scanning of the thorax, needle biopsy of the pleura, bronchoscopy, and sometimes pleuroscopy and open pleural biopsy. Indeed, invasive procedures are often justified because the criteria for diagnosing malignancy by morphological imaging (CT and MR imaging) are not specific and cytological examination of the pleural fluid has a low sensitivity. ${ }^{1}$

Positron emission tomography (PET) with ${ }^{18} \mathrm{~F}$-fluorodeoxyglucose (FDG) can accurately differentiate benign from malignant pulmonary abnormalities. Recent studies have shown its use in the evaluation of solitary pulmonary nodules and the staging of mediastinal nonsmall cell lung cancer. ${ }^{23}$
In this preliminary report we have investigated the ability of FDG-PET to differentiate between malignant and non-malignant pleural disease.

\section{Methods \\ PATIENT SELECTION}

Twenty five subjects of mean age 60 years (range 36-80) undergoing evaluation of pleural disease were enrolled in the study. Each subject was examined by chest radiography, CT scanning (PQ 2000 4th generation, Picker, Cleveland, Ohio, USA) and pleural fluid analysis. A PET study was performed on each subject before invasive procedures were carried out.

\section{PET STUDY}

PET was performed with an UGM Penn PET $240 \mathrm{H}$ scanner as previously described ${ }^{4}$ and the data were analysed by visual interpretation of coronal, sagittal, and transverse slices alone and by cross referencing. PET images were read independently by two nuclear physicians, who had knowledge of the standard chest radiograph only, by evaluation of the presence or absence of ${ }^{18}$ FDG uptake in the pleural space. When increased ${ }^{18}$ FDG uptake was observed, two levels were identified - moderate or intense.

\section{ANALYSIS OF DATA}

The diagnostic efficacy of PET-FDG imaging as a means of differentiating malignant from benign pleural lesions was evaluated by calculating the sensitivity, specificity, positive predictive value, and negative predictive value. For each parameter the $95 \%$ confidence intervals ( $95 \% \mathrm{CI})$ were given.

\section{Results}

Sixteen patients had malignant pleural disease and nine had benign pleural disease. Malignant diseases (13 metastatic lesions and three mesothelioma) were confirmed by analysis of pleural samples obtained by Abrams needle biopsy $(\mathrm{n}=4)$, thoracoscopy $(\mathrm{n}=11)$, or thoracotomy $(n=1)$. In nine patients the disease was considered benign: five parapneumonic effusions (diagnosed by plain radiographic follow up after thoracocentesis), one pleural fibroma (thoracotomy), one benign asbestos pleural disease (thoracoscopy), one benign neurofibroma (thoracotomy), and one tuberculous pleuritis (Abrams needle biopsy). At present the follow up for these benign pleural diseases is at least yearly with no signs of malignancy. 

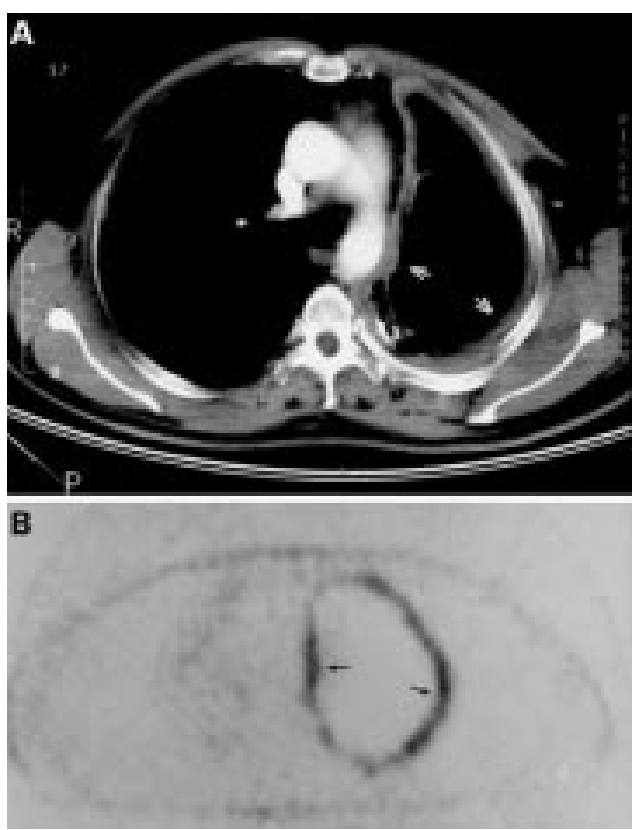

Figure 1 (A) CT scan and (B) FDG-PET imaging in a 52 year old man with sarcomatous type mesothelioma. The CT scan shows a circumferential and nodular pleural thickening (white arrows) and the PET scan is a transverse image showing intense FDG uptake within the pleural thickening (black arrows).

\section{PET IMAGING}

PET imaging correctly identified all malignant pleural diseases. All malignant pleural lesions showed a diffuse increase in ${ }^{18}$ FDG uptake

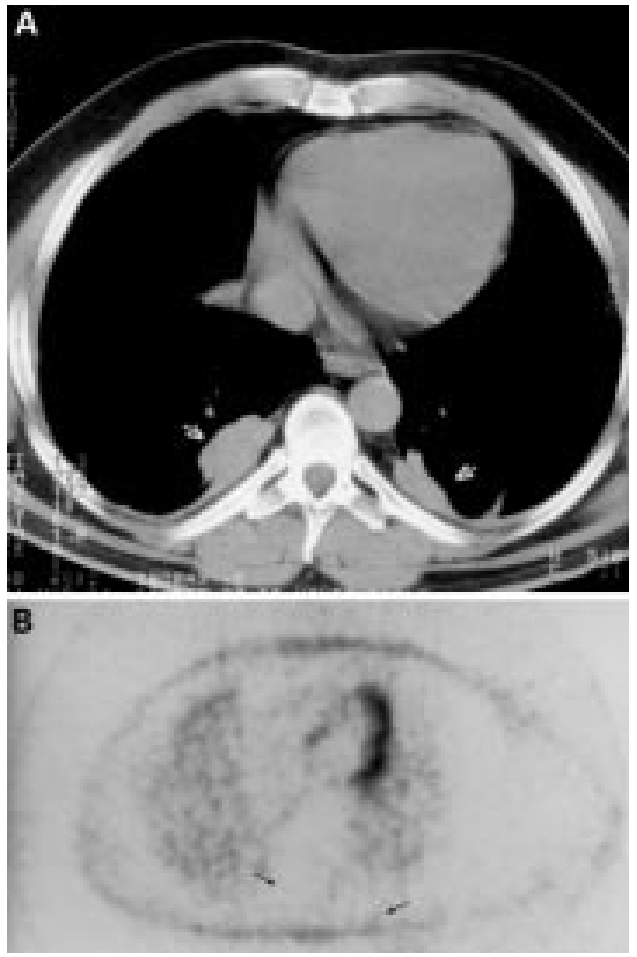

Figure 2 (A) CT scan and (B) FDG-PET imaging in a 40 year old man with benign asbestos pleural disease. The CT scan shows bilateral nodular pleural thickenings in paravertebral gutters (white arrows) and the PET scan is a transverse image showing the absence of FDG uptake within the posterior pleural thickenings (black arrows). Note the anterior FDG uptake by the heart. within the pleural thickening. The sensitivity of the method was $100 \%$ (95\% CI 79.4 to 100 ) with a negative predictive value of $100 \%(95 \%$ CI 59 to 100). The ${ }^{18}$ FDG uptake was intense in 14 cases and moderate in two. Figure 1 shows CT and FDG-PET scans in a patient with diffuse unilateral involvement of the pleura by a sarcomatous type mesothelioma.

PET imaging showed the absence of ${ }^{18} \mathrm{FDG}$ uptake within the pleura and correctly identified seven benign lesions. In two patients with benign pleural disease there was an increase in FDG uptake which was moderate and localised to the lateral pleural space; one patient had a parapneumonic effusion and the second a tuberculous pleuritis. Figure 2 shows CT and FDG-PET scans in a patient with benign pleural disease. The specificity of the FDGPET imaging was $78 \%$ (95\% CI 40 to 97 ) and the positive predictive value was $89 \%(95 \% \mathrm{CI}$ 65.3 to 98.6 ).

\section{Discussion}

It is easy to confirm the presence of a pleural abnormality by clinical examination and CT scanning of the thorax. However, the morphological patterns on CT scans alone cannot accurately differentiate between benign and malignant lesions. ${ }^{5}$ For this reason, an invasive procedure (thoracocentesis, needle biopsy, thoracoscopy, open pleural biopsy) is often required to establish the diagnosis. Little is known about the application of FDG-PET imaging in the management of pleural disease, ${ }^{6}$ but our preliminary results suggest that it may be useful in the evaluation of certain pleural diseases. Benign and malignant primary pleural tumours are rare and a "limited" thoracotomy is often required to establish the diagnosis. In our study the malignant or benign nature of these primary pleural abnormalities $(n=6)$ was correctly identified by this non-invasive technique. Metastatic pleural lesions are common but may be difficult to diagnose so that thoracoscopy, requiring admission to hospital for five days, is necessary. In our series there were 13 cases of metastatic pleural involvement. All showed a diffuse increase in ${ }^{18}$ FDG uptake which was intense in 11 cases and moderate in two. PET imaging with visual interpretation appears to be less conclusive in infectious pleural diseases; in our series we observed two such cases with moderate FDG uptake.

On the basis of these preliminary results we believe that the PET technique could reduce the number of open pleural biopsies and "limited" thoracotomies performed for benign pleural disease. In particular, if a patient has localised pleural thickening which does not exhibit ${ }^{18}$ FDG uptake, we think it is unnecessary to subject the patient to an invasive procedure as a first step. This will have to be confirmed in a larger study.

We do not routinely quantify our PET data because acquisition of transmission data is currently incompatible with whole body imaging as it would prolong the procedure by an hour or more, depending on the length of the scan. 
However, quantification of PET data, if possible, would allow precise definition of the degree of increased ${ }^{18}$ FDG uptake so that a needle biopsy could thus be performed in a region of intense uptake. In addition, it might assist in distinguishing an infective pleural process from metastatic disease.

In conclusion, our preliminary results show that FDG-PET could be an effective tool in the evaluation of pleural diseases. Further studies are needed to validate our preliminary descriptions and to develop quantification programmes.
1 Sahn S. Malignant pleural effusions. In: Fischman AP, ed. Pulmonary diseases and disorders. 2nd edn. New York: McGraw-Hill, 1988: 2159-70.

2 Dewan N, Gupta N, Redepenning L, Phalen J, Frick M Diagnostic efficacy of PET-FDG imaging in solitary pulmonary nodules. Chest 1993;104:997-1002.

3 Wahl R, Quint L, Greenough R, Meyer C, White R, Orringer $M$. Staging of mediastinal non-small cell lung cancer with FDG-PET, CT, and fusion images: preliminary prospective evaluation. Radiology 1994;191:371-7.

4 Bury T, Dowlati A, Paulus P, Corhay JL, Benoit T, Kayembe $\mathrm{JM}$, et al. Evaluation of the solitary pulmonary nodule by positron emission tomography imaging. Eur Respir $\mathcal{F} 1996$; positron emi 9 :410-4.

5 Leung A, Muller N, Miller R. CT in differential diagnosis of diffuse pleural disease. AfR 1990;154:487-92.

6 Bischof H, Knopp M, Bucher M, Manegold C, Van Kaick G, Drings P. Clinical application of FDG-PET imaging in management of malignant pleural mesothelioma. Eur Respir f 1993;6:140s 